\title{
Macrophage Polarization is Deregulated in Haemophilia
}

\author{
Lynn M. Knowles ${ }^{1}$ Daniela Kagiri ${ }^{1} \quad$ Martin Bernard ${ }^{1} \quad$ Eva C. Schwarz ${ }^{2}$ Hermann Eichler ${ }^{1}$ Jan Pilch ${ }^{1}$ \\ 1 Institute of Clinical Hemostaseology and Transfusion Medicine, \\ Saarland University Medical Center, Saarland University Faculty of \\ Medicine, Saarland University, Homburg, Germany \\ 2 Department of Biophysics, CIPMM, Saarland University Faculty of \\ Medicine, Saarland University, Homburg, Germany \\ Address for correspondence Jan Pilch, MD, Institute of Clinical \\ Hemostaseology and Transfusion Medicine, Saarland University \\ Medical Center, Ringstr. 52 / Geb. 1, D-66421 Homburg/Saar, \\ Germany (e-mail: jan.pilch@uks.eu).
}

Thromb Haemost 2019;119:234-245.

\begin{abstract}
Macrophages make important contributions to inflammation and wound healing. We show here that macrophage polarization is deregulated in haemophilia in response to macrophage colony-stimulating factor (M-CSF) and partially in response to granulocyte-macrophage colony-stimulating factor (GM-CSF). As a result, haemophilia macrophages exhibit a specific impairment of M-CSF-mediated functions involved in wound healing such as clot invasion and phagocytosis. Haemophilia monocytes express reduced amounts of the receptors for M-CSF and GM-CSF, which correlates with a failure to express tumour necrosis factor $\alpha(T N F \alpha)$ and CD163 in M-CSF-treated haemophilia macrophages and reduced expression of TNF $\alpha$ and CD206 after treatment with GM-CSF. Protein expression in response to M-CSF was regained with respect to CD163 and CD206 after embedding haemophilia monocytes in clotted plasma suggesting that a functioning coagulation system has positive effects on macrophage M2 polarization. Mimicking the functional deficits of haemophilia macrophages in normal macrophages was possible by adding leptin, which we found to be elevated in the blood of haemophilia patients, to a monocyte cell line. The increase of leptin occurred in conjunction with C-reactive protein in a body mass index-controlled cohort

Keywords

- haemophilia

- macrophage

- fibrin suggesting that haemophilia patients harbour chronic low-grade inflammation. Together, our data indicate that impaired clotting in haemophilia patients leads to increased inflammation and a deregulation in macrophage differentiation, which may explain the commonly observed deficits in wound healing and tissue regeneration.
\end{abstract}

\section{Introduction}

The response to tissue injury is typically marked by activation of the clotting cascade followed by recruitment of inflammatory cells and the initiation of tissue regeneration. ${ }^{1}$ In bleeding disorders, such as haemophilia, this process is impaired, resulting in protracted bleeding and prolonged wound healing. ${ }^{2}$ Bleeding complications in haemophilia are common in the musculoskeletal system with the majority of bleeding episodes being located in the large joints, which in turn cause inflammation of the affected joints thus resulting in haemophilic arthropathy. ${ }^{3,4}$ While the prophylactic substitution of coagulation factors is sufficient to substantially delay the onset of clinically apparent arthropathy, most if not all haemophilia patients ultimately develop joint disease even under treatment conditions that suppress clinically recognizable bleeding. ${ }^{5}$

Extravasation of red blood cells (RBCs) and the subsequent release of their iron content inside the joint is thought to be an important element of the molecular pathology of haemophilic arthropathy. ${ }^{6,7}$ This view is based on experimental data showing that exposing the synovia of mice to overwhelming amounts of ferrous iron can trigger an inflammatory reaction that is associated with the activation of the nuclear factor-KB pathway in synoviocytes and subsequent systemic release of inflammatory cyto-/chemokines. ${ }^{8}$ In line
License terms Stuttgart · New York 10.1055/s-0038-1676796 ISSN 0340-6245.
DOI https://doi.org/
June 12, 2018

accepted after revision

November 10, 2018

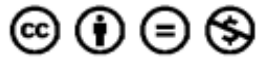


with this, it has been demonstrated that macrophages in joints from patients with haemophilic arthropathy express a wide array of inflammatory mediators suggesting that monocytic cells differentiate after their recruitment from the blood circulation to actively contribute to inflammatory joint disease. ${ }^{9} \mathrm{M} 2$ macrophages, on the other hand, which are specifically equipped to sequester tissue debris including RBCs and haemoglobin, have been found to be under-represented in haemophilic target joints. ${ }^{10}$

Coagulation factor deficiencies affect monocyte function not only through clinical or sub-clinical trauma, but also as a direct result of clotting impairment. ${ }^{11,12}$ Specific examples for the coupling of inflammation and clotting include the contribution of tissue factor on activated monocytes to the aetiology of venous thromboembolism, the stimulating effects of thrombin on monocyte transmigration as well as the impaired endothelial function during sepsis. ${ }^{13-15}$ In addition, monocytes require adhesive interactions with the clotting end product fibrin, which depending on the tissue context promote inflammation or wound healing. ${ }^{12,16}$ As such, it has been shown that monocyte recruitment is delayed in mice with haemophilia B and that this delay correlates with impaired wound healing. ${ }^{2}$ Based on these data, we aim to test if macrophage function is deregulated in patients with haemophilia and whether this affects functions such as RBC removal and clot invasion.

\section{Materials and Methods}

\section{Human Subjects}

Patients were recruited from our certified European Haemophilia Comprehensive Care Centre; healthy blood donors from our blood donation service. Approval was received from the local ethics committee (Kenn-Nr. 73/14, Ethik-Kommission Ärztekammer Saarland) and blood was drawn after receiving informed consent. Patients as well as donors were instructed not to skip meals before blood drawing.

\section{Cell Purification and Culture}

Peripheral blood mononuclear cells (PBMCs) were isolated from citrated blood using Lymphocyte Separation Medium 1077 according to the manufacturer's specifications (Promocell). Monocytes were separated from the PBMC population by allowing the cells to adhere to tissue culture dishes for 2 hours in the presence of serum-free media. Lymphocytes, which do not adhere to the culture dishes, were removed by aspiration. To attain a monocyte suspension, we purified monocytes from PBMCs using the Dynabeads Untouched Human Monocytes Kit (Invitrogen). This isolation method was used for all subsequent assays involving phenotyping, filopodia measurement, phagocytosis and three-dimensional (3D) cell culture.

For 3D cell culture, Dynabeads-purified primary monocytes were mixed with human plasma (US Biological) in the presence of $3 \mathrm{mM} \mathrm{CaCl}_{2}$ to generate clotted plasma. Clotting was induced with $3 \mathrm{U} / \mathrm{mL}$ thrombin (Sigma-Aldrich), and $15 \mu \mathrm{L}$ suspensions were pipetted onto tissue culture plates and inverted at room temperature for 10 minutes to solidify. In addition, Dynabeadspurified monocytes were used to confirm polarization on plastic and test phagocytosis as well as the expression of differentiation markers. Independent of the isolation method, monocytes were incubated with RPMI media supplemented with L-glutamine, penicillin and streptomycin, and 10\% foetal bovine serum (FBS) at $37^{\circ} \mathrm{C}$ under a humidified, $5 \% \mathrm{CO}_{2}$ atmosphere. PBMC-derived monocytes were treated with $125 \mathrm{ng} / \mathrm{mL}$ granulocyte-macrophage colony-stimulating factor (GM-CSF) (Cellgenix) or 100 $\mathrm{ng} / \mathrm{mL}$ macrophage colony-stimulating factor (M-CSF) (Miltenyi Biotec) to generate M1 and M2 macrophages, respectively. Where indicated, M-CSF- or GM-CSF-treated macrophages were further activated with $20 \mathrm{ng} / \mathrm{mL}$ interferon $\gamma($ IFN- $\gamma$ ) (PeproTech) and $100 \mathrm{ng} / \mathrm{mL}$ lipopolysaccharides (LPS) (SigmaAldrich) or $20 \mathrm{ng} / \mathrm{mL}$ interleukin 4 (IL-4) (Cellgenix). THP-1 monocytes were purchased from DSMZ and propagated in RPMI media containing $10 \% \mathrm{FBS}$ and $0.05 \mathrm{mM} \beta$-mercaptoethanol (Carl Roth). THP-1 monocytes were differentiated to M0 macrophages by treatment with $25 \mathrm{nM}$ phorbol 12-myristate 13acetate (Sigma-Aldrich) for 24 hours, washed and incubated with normal media for 24 hours and then incubated with M-CSF for 96 hours. THP-1 monocytes were pre-incubated with recombinant human leptin (500 ng/mL; R\&D Systems) for 96 hours prior to the addition of M-CSF.

\section{Morphological Analysis}

To assess cell polarization, macrophages attached to plastic or embedded in clots were analysed at designated times by bright field microscopy (Zeiss Primo Vert). To this end, we counted cells based on their shape in an average of at least three distinct optical fields in at least five different samples. Macrophages with a spread, elongated phenotype were classified as polarized, while cells that remained round (with or without rudimentary pseudopodes) were counted as non-polarized. Results show the number of polarized cells as per cent of the total cell number per microscopy field. As an additional measure of macrophage polarization, we determined the elongation index (cell length/cell width) using the Image J software.

\section{Fluorescence Microscopy}

Macrophages were fixed in $4 \%$ paraformaldehyde, permeabilized with $0.2 \%$ triton $\times 100$ and incubated with anti-CD68 (Abcam), anti-tumour necrosis factor $\alpha$ (TNF $\alpha)$ (Dianova), anti-CD206 (AbD Serotec), anti-CD163 (AbD Serotec) or isotype control, followed by incubation with Alexa Fluor 488-conjugated secondary antibody (Invitrogen) and analysed using a fluorescence microscope (Nikon H550L). To visualize filopodia and nuclei, cells were stained with Alexa Fluor 546-phalloidin (Invitrogen) and 4',6-diamidino-2-phenylindole, respectively. Digitized images were processed with Adobe Photoshop. Experiments were performed and analysed by two laboratory members independently to guarantee reproducibility of the evaluation criteria.

\section{Phagocytosis Assay}

Latex bead phagocytosis was examined using a commercially available Phagocytosis Assay Kit (Cayman Chemical). Briefly, macrophages were incubated for 1 hour with latex beads coated with fluorescein isothiocyanate (FITC)-labelled rabbit 
immunoglobulin $\mathrm{G}$ at $37^{\circ} \mathrm{C}$, then washed with phosphatebuffered saline (PBS) and incubated for 1 minute with Trypan blue to quench surface FITC fluorescence. To assess RBC phagocytosis by macrophages, whole blood was diluted with equal part PBS containing $5 \mathrm{mM}$ ethylenediaminetetraacetic acid, centrifuged at $300 \times g$ to remove the plasma and buffy coat fractions and the resulting RBCs were washed three times with PBS. RBCs $\left(5 \times 10^{8}\right.$ cells $\left./ \mathrm{mL}\right)$ were then labelled with $50 \mu \mathrm{M}$ Cell Tracker Green CMFDA (Invitrogen) in the dark for 30 minutes at $37^{\circ} \mathrm{C}$, centrifuged to pellet and opsonized with $100 \mu \mathrm{g} / \mathrm{mL}$ anti-human RBC Antibody (Rockland) for 30 minutes. Suspensions were centrifuged, the pellet was re-suspended in $2.5 \mathrm{~mL}$ serum-free RPMI media and $50 \mu \mathrm{L}$ was added to designated wells in 8-well chamber slides containing $200 \mu \mathrm{L}$ media. After 1 hour, cultures were washed with sterile $\mathrm{dH}_{2} \mathrm{O}$ to lyse non-phagocytosed RBCs, fixed and analysed by fluorescence microscopy.

\section{Flow Cytometry}

Fluorescent-labelled antibodies against CD14 (Anti-CD14 FITC, BD Biosciences), CD16 (Anti-CD16 PE, BD Biosciences), C-C chemokine receptor type 2 (CCR2) (Anti-CCR2 APC, R\&D Systems), GM-CSF receptor (GM-CSFR) (Anti-CD116 APC, Miltenyi Biotec), colony stimulating factor 1 receptor (CSF1-R) (Anti-CSF1-R APC, R\&D Systems), Tie2 (Anti-Tie2 APC, R\&D Systems) and CD163 (Anti-CD163 APC, Miltenyi Biotec) were purchased alongside with the appropriate control antibodies. Whole blood $(100 \mu \mathrm{L})$ was co-stained with CD14 FITC along with the APC- or PE-conjugated antibodies for 20 minutes at room temperature in the dark. RBCs were subsequently lysed with BD FACS Lyse solution for 10 minutes, suspensions were centrifuged at $300 \times g$ for 5 minutes and pellets were washed once with BD Cell Wash. Antibody binding was analysed using a FACSCalibur flow cytometer (BD Biosciences) equipped with CellQuest software and analysed using FlowJo. The specific forward/sideward scatter characteristics of leukocytes allowed us to clearly identify the monocyte population.

\section{Protein Array}

Platelet-poor plasma was isolated from citrate blood by centrifugation at 3,000 revolutions per minute for 20 minutes at room temperature. Cytokine levels in plasma were examined using the Proteome Profiler Human XL Cytokine Array Kit (102 human cytokines, R\&D Systems) according to the manufacturer's recommendations. Briefly, the antibody arrays were incubated with $100 \mu \mathrm{L}$ plasma overnight at $4^{\circ} \mathrm{C}$. The next day, the arrays were washed, incubated with biotinylated antibody cocktail for 1 hour, streptavidin-horseradish peroxidase (HRP) for 30 minutes and visualized by enhanced chemiluminescence using the GeneGnome HR imaging system (SynGene) with GeneSnap software. Cytokine expression was quantitated in Image J using the Protein Array Analyzer.

\section{Cytokine Enzyme-Linked Immunosorbent Assay}

Platelet-poor plasma was analysed for the expression of leptin and C-reactive protein (CRP) using the Quantikine ELISA Kit from R\&D Systems. In brief, leptin and CRP precoated micro-plates were incubated with plasma for 2 hours, washed to remove unbound plasma constituents, incubated for 1 hour with human leptin or CRP HRP-linked monoclonal antibody, washed and developed by enhanced luminescence on a plate reader (Tecan SPARK) at $450 \mathrm{~nm}$ with a correction set to $540 \mathrm{~nm}$.

\section{Statistical Analysis}

Significance was determined using Student's two-tailed $t$ test or one-way analysis of variance followed by the post hoc Tukey's multiple comparisons test (GraphPad Prism 5). Treatment differences with a two-sided $p$-value of $<0.05$ were considered significantly different. Error bars show mean \pm standard error of the mean.

\section{Results}

\section{Haemophilia Patient Cohort}

Between 2014 and 2017, we enrolled into our study 48 adult male patients with haemophilia A or B that visited our outpatient clinic for routine testing ( - Table 1). At enrolment, the patients were in average 36 years of age and had a body mass index (BMI) of close to $28 \mathrm{~kg} / \mathrm{m}^{2}$. Thirty-seven patients

Table 1 Description of the patient cohort

\begin{tabular}{|c|c|}
\hline Characteristic & $\begin{array}{l}\text { All patients } \\
(n=48)\end{array}$ \\
\hline \multicolumn{2}{|l|}{ Bleeding disorder } \\
\hline Haemophilia A & $41(85)$ \\
\hline Haemophilia B & $7(15)$ \\
\hline \multicolumn{2}{|l|}{ Baseline coagulation factor activity } \\
\hline FVIII/IX $<1 \%$ & $37(77)$ \\
\hline On prophylaxis & $30(63)$ \\
\hline Average coagulation activity (range) & $11.9(0.3-77)$ \\
\hline FVIII/IX 1-15\% & $10(21)$ \\
\hline On prophylaxis & $1(2)$ \\
\hline Average coagulation activity (range) & $10.6(2-15)$ \\
\hline $\mathrm{FVIII} / \mathrm{IX}>15 \%$ & $1(2)$ \\
\hline On prophylaxis & 0 \\
\hline Average coagulation activity & 25 \\
\hline \multicolumn{2}{|l|}{ Complications } \\
\hline Bleeding $<3$ months & $27(56)$ \\
\hline Arthropathy & $22(46)$ \\
\hline Chronic infections (HBV, HCV, HIV) & $15(31)$ \\
\hline \multicolumn{2}{|l|}{ Treatment } \\
\hline Recombinant & $30(63)$ \\
\hline Plasmatic & $18(37)$ \\
\hline Age (range) & $36(18-68)$ \\
\hline Body mass index (range) & $27.7(18.7-38.0)$ \\
\hline
\end{tabular}

Abbreviations: FVIII/IX, factor VIII/IX; HBV, hepatitis B virus; HCV, hepatitis $C$ virus; HIV, human immunodeficiency virus. Note: Values are $n(\%)$ unless otherwise indicated. 
(77\%) had severe haemophilia (residual factor VIII [FVIII]/FIX activity $<1 \%$ ), of which $30(63 \%)$ received prophylactic treatment either with a recombinant or plasmatic coagulation factor concentrate. Consequently, the effective activity of coagulation FVIII or FIX in patients with severe haemophilia was at the time of blood drawing nearly indistinguishable to the patients with moderate to mild haemophilia (11.9 vs. 10.6\%). Moreover, just under half of the patients enrolled had clinical signs of arthropathy with at least one affected joint located at either the lower (ankle, knee, hip) or upper extremity (shoulder, elbow). The diagnosis of arthropathy was based on orthopaedic physical examination, the history of a target joint or past orthopaedic procedures (prosthetic joint replacement, synovectomy). Approximately one-half of the patients had bleeding events in the last 3 months and one-third of the patients were registered for having contracted chronic hepatitis B, hepatitis $C$ or human immunodeficiency virus (HIV). For controls, we randomly recruited male blood donors ( $n=60$; age, 35.8 years, range: $21-65$ years; BMI, 27.0, range: 22.3-37.6) from our blood donation centre at Saarland University Hospital.

\section{Haemophilia Monocytes Exhibit Defective Polarization}

Monocytes utilize adhesive interactions with the extracellular matrix to migrate and invade in response to wounding and inflammation. ${ }^{17}$ In addition, adhesion and cytoskeletal remodelling are major determinants of signal transduction and gene expression during macrophage differentiation. ${ }^{18}$ These changes can be measured ex vivo as monocytes begin to spread and undergo a typical shape change from round to elongated and polarized in response to M-CSF-containing differentiation media. ${ }^{19}$ To study monocyte to macrophage differentiation, we isolated monocytes from the peripheral blood of two patients, one with mild haemophilia A (residual FVIII activity $=5-10 \%$ ), the other with severe haemophilia B (residual FIX activity $<1 \%$ ), as well as four healthy blood donors and monitored cell spreading in response to M-CSF as well as GM-CSF by phase contrast microscopy. In addition, slides were stained with antibody against the monocyte/macrophage marker CD68 and inspected by fluorescence microscopy, which confirmed a $>90 \%$ pure culture 7 days after isolating monocytes with the Dynabeads Untouched kit (-Fig. 1A; - Supplementary Fig. S1A, available
A
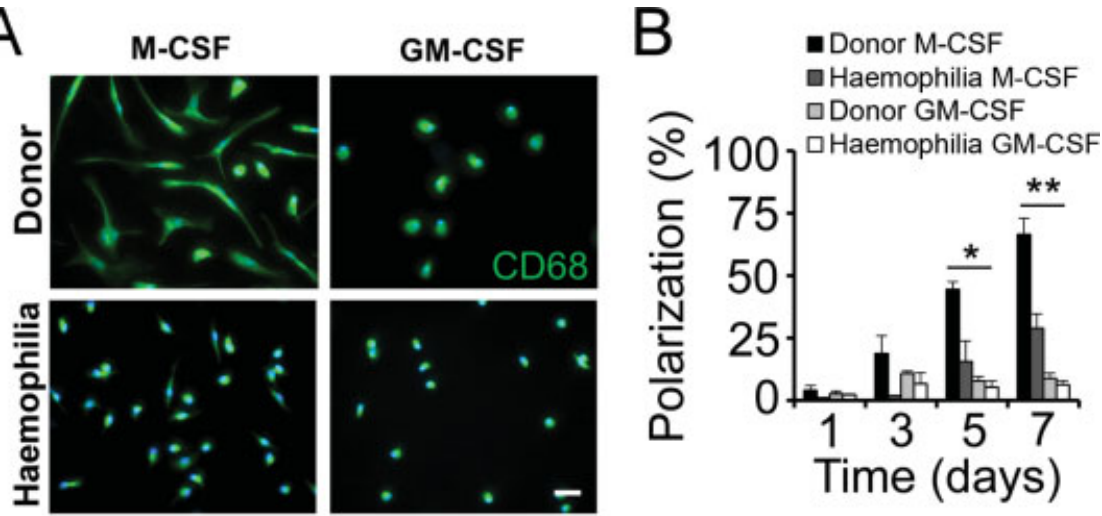

C

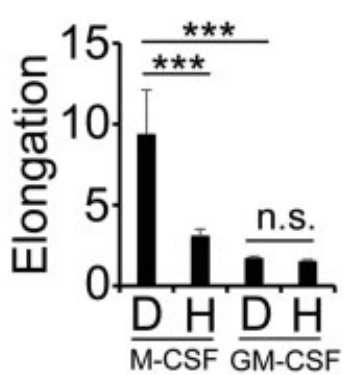

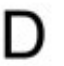

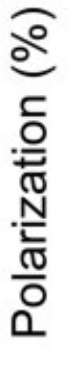

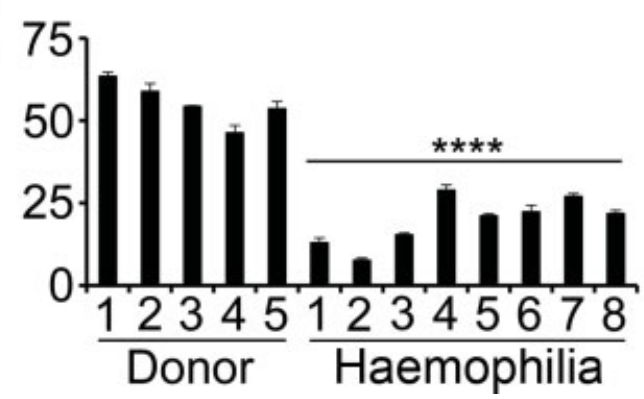

Fig. 1 Haemophilia macrophages exhibit defective polarization. (A) Monocytes purified from peripheral blood mononuclear cells (PBMCs) of healthy blood donors $(n=4)$ and haemophilia patients $(n=2)$ using Dynabeads were incubated for 7 days with 100 ng/mL macrophage colonystimulating factor (M-CSF) or $125 \mathrm{ng} / \mathrm{mL}$ granulocyte-macrophage colony-stimulating factor (GM-CSF) on cell culture plastic, then fixed and stained with anti-CD68/Alexa 488 anti-mouse immunoglobulin G (IgG) (green). Nuclei are stained with 4',6-diamidino-2-phenylindole (DAPI) (blue). Representative fluorescence microscopy images are shown. (B) Phase contrast images of purified monocytes from donors and haemophilia patients cultured on plastic were scored for cell polarization (polarized cells as percentage of total cells) over time. (C) Cell elongation (elongation index as ratio of length/width) was assessed in donor (D) and haemophilia macrophages (H) after 7 days of cell culture in M-CSF or GM-CSF. (D) PBMCs from eight haemophilia patients and five blood donors were allowed to attach to plastic, washed to remove unbound lymphocytes as well as granulocytes, then treated with M-CSF and finally analysed for macrophage polarization after 7 days. ${ }^{*} p<0.05$, ${ }^{* *} p<0.01,{ }^{* * *} p<0.001,{ }^{* * * *} p<0.0001$, n.s., non-significant. Scale bar, $20 \mu \mathrm{m}$. 
in the online version). Both fluorescence and phase contrast microscopy demonstrated that addition of M-CSF induced rapid spreading in donor macrophages, which exhibited an elongated, polarized phenotype in $>60 \%$ of cells after 7 days of treatment (-Fig. 1A, B). Treatment of macrophages with the pro-inflammatory growth factor GM-CSF also induced spreading, but in contrast to M-CSF only a small fraction of the macrophages polarized. Accordingly, the elongation index (cell length/cell width) was significantly higher after treatment with M-CSF compared with GM-CSF ( - Fig. 1C). Although M-CSF promoted polarization and elongation in the majority of donor macrophages, it was much less effective in mediating a similar shape change in macrophages from a panel of haemophilia patients, which broadly represent our patient cohort in terms of treatment (on-demand vs. prophylaxis), bleeding ( $<3$ month) and co-morbidities (arthropathy, hepatitis/HIV) (- Fig. 1D; - Supplementary Fig. S1B; - Supplementary Table S1, available in the online version). The observed changes appear to be connected to the clotting deficiencies as we detected a significant increase of macrophage polarization as well as elongation after the treatment with thrombin in combination with M-CSF (-Supplementary Fig. S1C and S1D, available in the online version). Together, our morphological analysis revealed a macrophage phenotype in haemophilia that is distinctly different from normal control macrophages and most pronounced after differentiation with M-CSF.

\section{Macrophages from Haemophilia Patients are Unable to Invade Blood Clot}

Blood clotting generates a mechanical tissue barrier after wounding and as such controls the recruitment of monocytes that are potentially involved in RBC phagocytosis and wound healing. ${ }^{2}$ Cell invasion and phagocytosis depend on the generation of filopodia, which could be visualized as finger-like actin-rich extensions on the cell surface of M-CSFas well as GM-CSF-treated donor macrophages (-Fig. 2). Haemophilia macrophages, in contrast, were only able to generate truncated filopodia or lacked the ability to generate filopodia altogether. To determine the capacity of haemophilia macrophages to invade blood clot, we embedded monocytes from two patients with haemophilia A and B in clotted plasma and assessed podosome formation as well as cell elongation as a measure of cell invasion. In response to M-CSF, podosome formation was detectable in donor macrophages 48 hours after embedding and rose to almost $80 \%$ podosome-positive cells by day 7 ( - Fig. 3A, B). Overall, this process was more effective in the presence of M-CSF than GM-CSF, which was superior to untreated monocytes at the early time points but later on had no additional effects on macrophage invasion (-Fig. 3B). Podosome formation and the resulting elongation of clot-embedded haemophilia macrophages, on the other hand, lagged behind corresponding values achieved with donor macrophages whether the macrophages were treated with M-CSF, GM-CSF or left untreated (-Fig. 3A, C, D). Together, these data show that $\mathrm{M}-\mathrm{CSF}$ is a potent pro-invasive stimulus for healthy macrophages. Haemophilia macrophages, on the other hand, lack the capacity to generate filopodia and infiltrate clotted
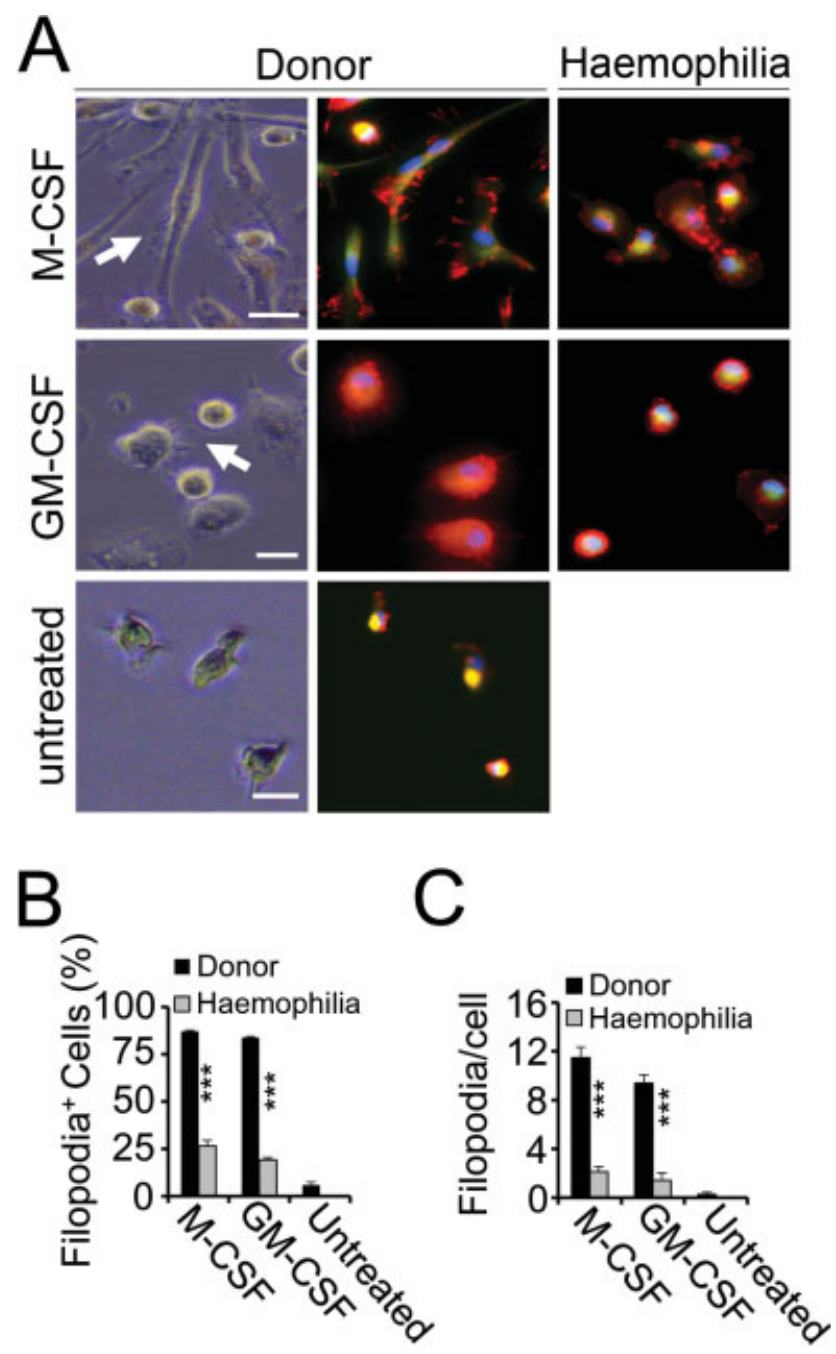

Fig. 2 Macrophages from haemophilia patients are unable to generate filopodia. (A) Representative phase contrast and fluorescence microscopy images of macrophages from healthy donors (left, middle) and haemophilia patients (right) after Dynabeads isolation and 7 days of cell culture on plastic in the presence of macrophage colony-stimulating factor (M-CSF) (top) or granulocyte-macrophage colony-stimulating factor (GM-CSF) (middle) compared with untreated monocytes (bottom). Fluorescence microscopy shows merged images after staining for the macrophage marker CD68 (green), F-actin (red) and nuclei (blue). Arrows indicate filopodia on donor macrophages. (B, C) Microscopy fields were analysed for filopodia-positive macrophages (B; per cent of total) and average number of filopodia per cell (C). Representative data from 2 patients and 2 donors are shown.

${ }^{* * *} p<0.001$. Scale bar, $20 \mu \mathrm{m}$.

plasma suggesting that the recruitment of monocytes to wounds could be diminished in haemophilia.

\section{Haemophilia Macrophages have Deficits in Phagocytosis}

Macrophages promote wound healing as they clear tissue debris, neutrophils and extravasated RBCs through phagocytosis. Interestingly, macrophage phagocytosis and polarization are governed by overlapping cytoskeletal mechanisms. ${ }^{20}$ To systematically analyse the correlation between the two functions, we assessed cell spreading in conjunction with phagocytosis in cytokine-treated macrophages after addition of fluorescence-conjugated latex beads. Using fluorescence 

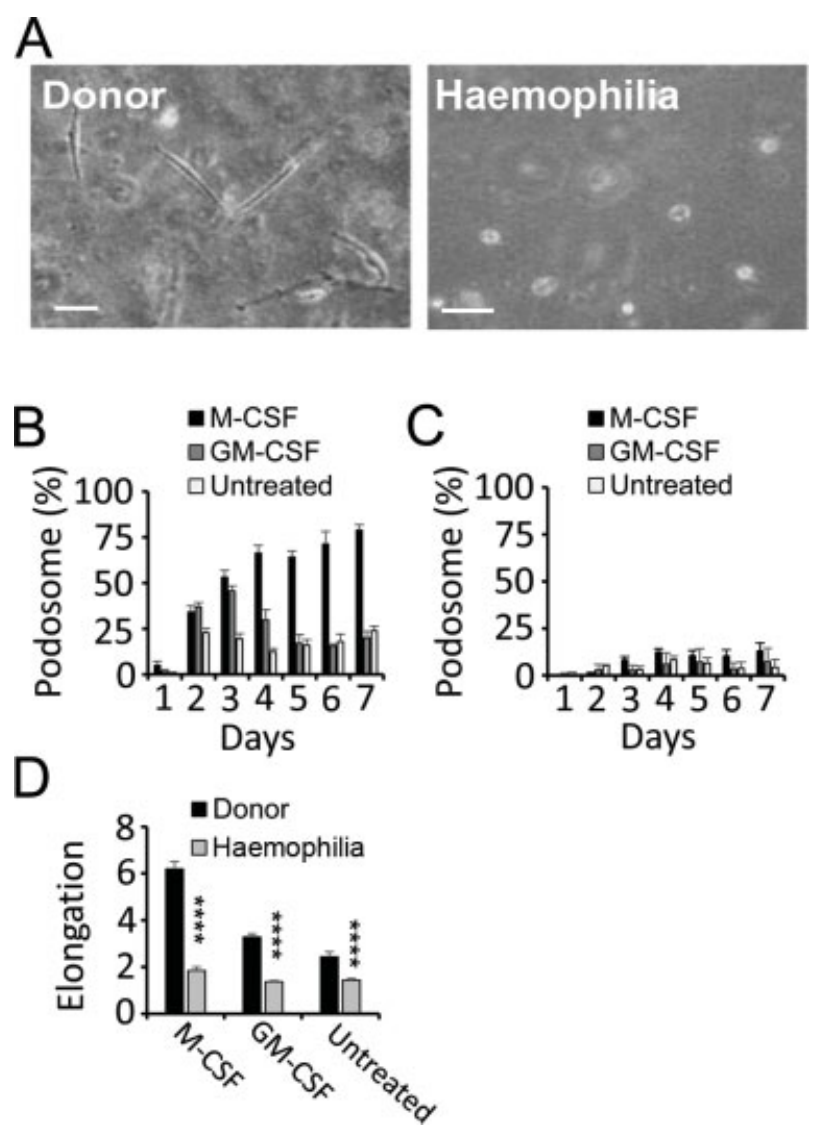

Fig. 3 Macrophages from haemophilia patients are unable to invade blood clot. (A) Representative phase contrast images of Dynabeads-purified monocytes from healthy donors (left; $n=2$ ) and haemophilia patients (right; $n=2$ ) 7 days after embedding in clotted plasma and treatment with macrophage colony-stimulating factor (M-CSF). (B, C) Monocytes from donors (B) and haemophilia patients (C) embedded in clotted plasma in the presence of M-CSF, GM-CSF or without treatment were scored for polarized, podosome-positive macrophages as per cent of total over time. (D) Elongation of macrophages as ratio of length/width was assessed 7 days after embedding in clotted plasma. ${ }^{* * *} p<0.0001$. Scale bar, $50 \mu \mathrm{m}$.

microscopy, we detected strong phagocytosis after an 8-day treatment of M-CSF with or without IL-4 in donor macrophages, which at the same time responded to M-CSF with extensive polarization as shown by phase contrast microscopy ( - Fig. 4A, B). In keeping with this symmetry, phagocytosis and cell polarization were equally reduced in M-CSF-treated donor macrophages after addition of IFNY/LPS and nearly abolished in GM-CSF-treated donor macrophages with or without IFNY/ LPS. While polarization and phagocytosis in macrophages from healthy individuals was strongest after treatment with $\mathrm{M}$-CSF alone, the cytokine was significantly less effective in mediating the uptake of latex beads in macrophages from two patients with haemophilia A and B (-Fig. 4C). Moreover, this was also the case when we tested phagocytosis of material relevant to the pathology of haemophilic arthropathy such as RBCs, which were taken up more efficiently by donor than by haemophilia macrophages (-Fig. 4A, C). We, therefore, conclude that haemophilia macrophages have a diminished capacity to sequester cell and tissue debris and that this may have important implications for wound healing after joint bleeding.
A
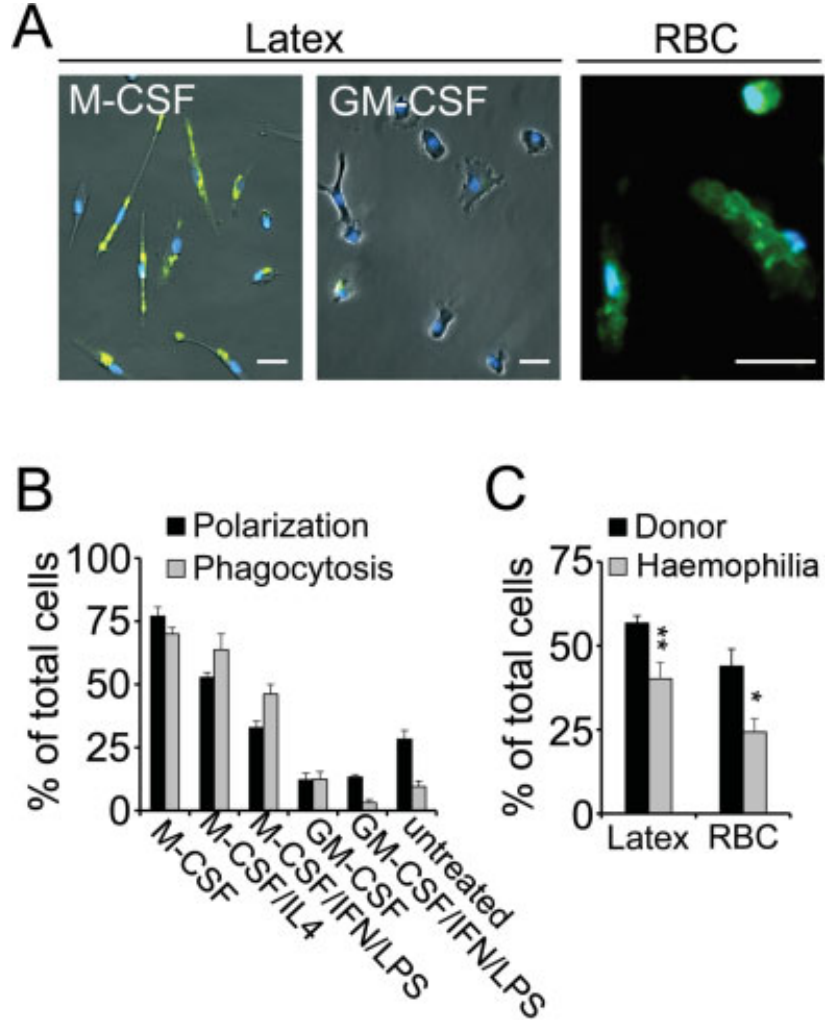

Fig. 4 Haemophilia macrophages have deficits in phagocytosis. (A) Monocytes from healthy blood donors were isolated with Dynabeads and incubated for 8 days with $100 \mathrm{ng} / \mathrm{mL}$ macrophage colony-stimulating factor (M-CSF) or $125 \mathrm{ng} / \mathrm{mL}$ granulocyte-macrophage colony-stimulating factor (GM-CSF) on cell culture plastic, then incubated with fluorescein-labelled latex beads (green) or red blood cells (RBCs; green; M-CSF only), fixed and stained with 4',6-diamidino-2-phenylindole (DAPI) to visualize nuclei (blue). Representative fluorescence microscopy images are shown. (B) Macrophages treated with M-CSF, GM-CSF or vehicle were scored for cell polarization and phagocytosis as per cent of total cells per microscopy field on day 8. Interleukin 4 (IL-4) and interferon y (IFNy)/lipopolysaccharides (LPS) were added on day 7 where indicated. (C) Phagocytosis of fluorescence-labelled latex beads or red blood cells (RBCs) in donor and haemophilia macrophages after 8 days of culture in M-CSF. Representative data from 2 patients and 2 donors are shown. ${ }^{*} p<0.05,{ }^{* *} p<0.01$. Scale bar, $20 \mu \mathrm{m}$.

\section{Macrophages from Haemophilia Patients are Unresponsive towards the Mitogenic Effects of M-CSF and GM-CSF}

Our data implicate a functional shift of haemophilia monocytes away from regenerative macrophage functions such as clot invasion and RBC phagocytosis. Based on the observation that haemophilia macrophages were not fully responsive towards M-CSF and GM-CSF in these assays, we performed flow cytometry to determine the expression of their respective receptors. While analysing CD14-positive monocytes from freshly drawn whole blood of 15 haemophilia patients and an equal number of healthy blood donors, we detected a substantial reduction of CSF1-R and to a lesser degree of GM-CSFR (- Fig. 5A-C, G; - Supplementary Table S2, available in the online version). In addition, haemophilia monocytes exhibited a significant reduction of two bona fide markers of tissue regeneration, namely, the haemoglobin scavenger receptor CD163 and the receptor tyrosine kinase Tie2 (-Fig. 5D, E, G). The expression of 

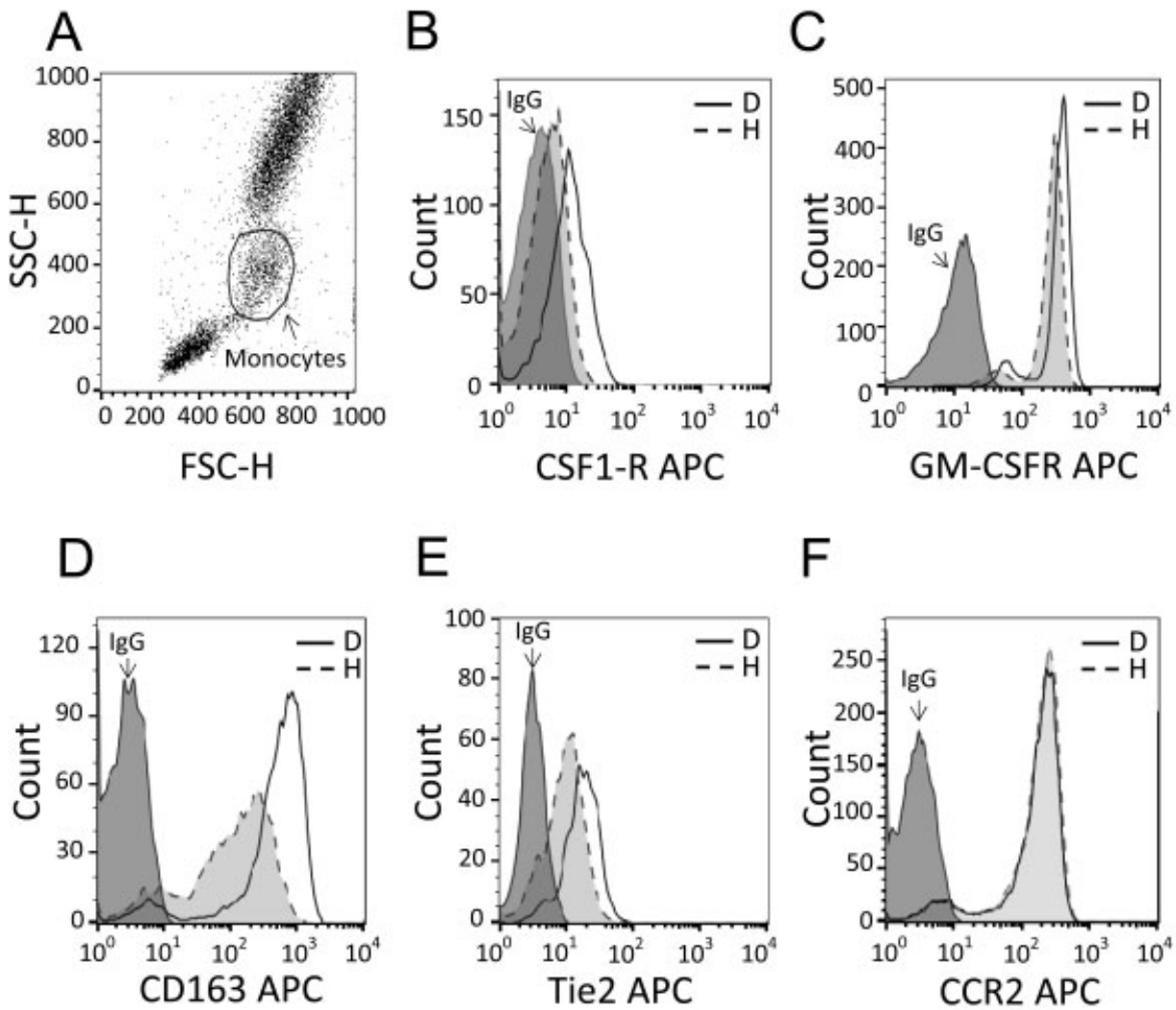

$\mathrm{F}$
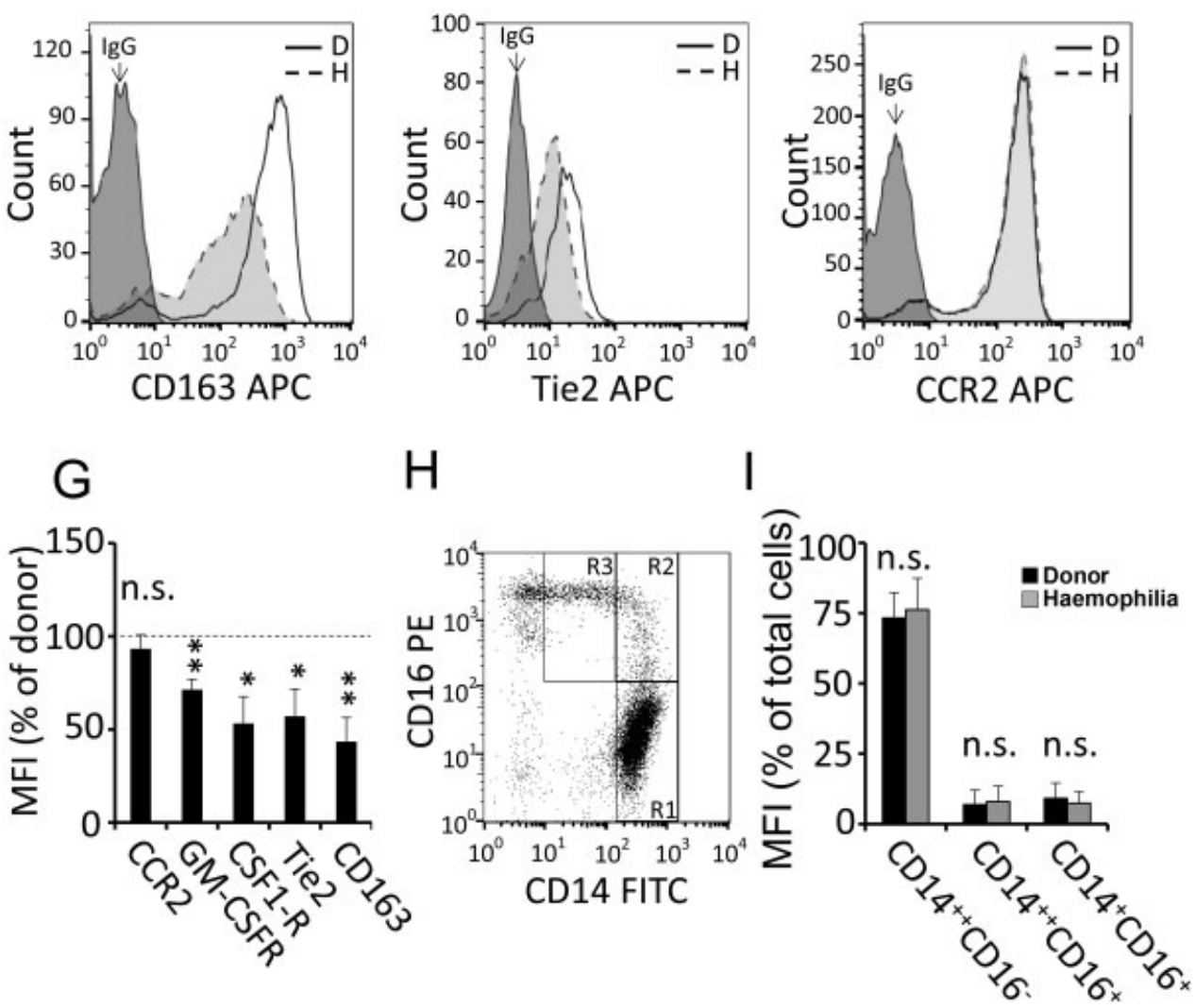

Fig. 5 Monocytes from haemophilia patients expressed reduced levels of M2 macrophage markers. (A) Representative flow cytometry profile of peripheral blood leukocytes examined by forward and side scatter properties to identify the monocyte population. (B-F) CD14 ${ }^{+}$monocytes from 15 haemophilia patients and 15 healthy blood donors were analysed for cell surface expression of colony stimulating factor 1 receptor (CSF1-R) (B; $n=6)$, granulocytemacrophage colony-stimulating factor receptor (GM-CSFR) (C; $n=3)$, CD163 (D; $n=3)$, Tie2 (E; $n=7)$ and C-C chemokine receptor type 2 (CCR2) (F; $n=8)$ using flow cytometry on blood isolated from haemophilia patients (H, dashed line, light grey histogram) compared with healthy blood donors (D, solid line, open histogram). Representative images are shown. Immunoglobulin G (lgG), dark grey histogram. (G) Results shown as per cent geometric mean fluorescence intensity on haemophilia monocytes compared with healthy blood donors. $(\mathrm{H}, \mathrm{I})$ Percentage of monocytes co-expressing CD14 and CD16 were

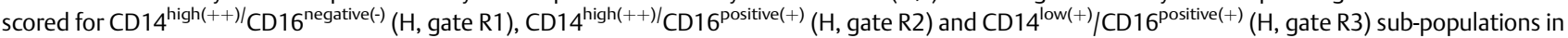
blood drawn from haemophilia patients $(n=9)$ compared with healthy blood donors $(n=11) .{ }^{*} p<0.05,{ }^{* *} p<0.01$, n.s., non-significant.

the chemokine receptor CCR2, on the other hand, was unchanged, which is in line with our data showing that there was no shift from $\mathrm{CD} 14^{\text {high }} \mathrm{CD} 16^{\text {negative }}$ to $\mathrm{CD} 14^{\text {low }} \mathrm{CD} 16^{\text {positive }}$ monocytes in the two cohorts ( $\mathbf{- F i g . ~ 5 F - I ) . ~ T o ~ f o l l o w ~ u p ~ o n ~ t h e ~}$ flow cytometry results, we used fluorescence microscopy to assess the induction of macrophage differentiation markers in purified monocytes in one haemophilia $A$ and one haemophilia B patient after 7 days in conventional two-dimensional (2D) culture on plastic plates compared with 3D culture in plasma clot. In plastic attached donor macrophages, we detected significant staining for TNF $\alpha$ in combination with CD206 after treatment with GM-CSF and to a somewhat lesser extent in combination with CD163 after treatment with M-CSF ( - Fig. 6A-C). Notably, the function to express macrophage differentiation markers on plastic was impaired in GM-CSFtreated haemophilia monocytes and completely lost when 
haemophilia monocytes were cultured in the presence of MCSF. Moreover, the inability of M-CSF to induce M2 markers in haemophilia macrophages was reversed after embedding in clotted plasma derived from healthy donors, which helped maintain CD163 on haemophilia macrophages, supported de novo expression of CD206 on donor as well as haemophilia macrophages and up-regulated TNF $\alpha$ on haemophilia macrophages after it was down-regulated in clot-embedded donor macrophages ( $\mathbf{F i g}$. 6A, D). Together, these data show that haemophilia monocytes display reduced expression of CSF1-R and GM-CSFR, which results in impaired macrophage differentiation in conjunction with a significant loss of M2 characteristics in 2D cultures. Significantly, these changes can be largely prevented by surrounding monocytes with clot from nonhaemophilic donors.

Leptin Restrains Macrophage Function in Haemophilia Our data demonstrate functional deficits of haemophilia macrophages, which can be traced back to a diminished response of monocytes towards differentiating stimuli. To identify possible underlying mechanisms, we employed a cytokine array to analyse the expression of $>100$ inflammatory and anti-inflammatory proteins in citrated plasma from one patient with haemophilia $A$ and in one patient with haemophilia B compared with two healthy blood donors. Using this kind of a scaled proteomics approach, we identified leptin (haemophilia/donor, 6.0-fold) as well as Fas ligand (haemophilia/donor, 1.9-fold) as the only two cytokines upregulated in both patients (-Fig. 7A). Moving forward, we focused on leptin, which caused a significant reduction of macrophage spreading as well as clot invasion when it was added to human THP-1 monocytes 96 hours prior to differentiation with M-CSF ( - Fig. 7B, C). Moreover, we recognized a significant drop in the expression of the macrophage differentiation markers TNF $\alpha$ and CD163 by fluorescence microscopy after pre-treatment with leptin and subsequent stimulation with M-CSF ( - Fig. 7D). Together, these data demonstrate that leptin is able to induce functional and phenotypical changes in an immortalized monocyte cell line that we otherwise detect in haemophilia macrophages.
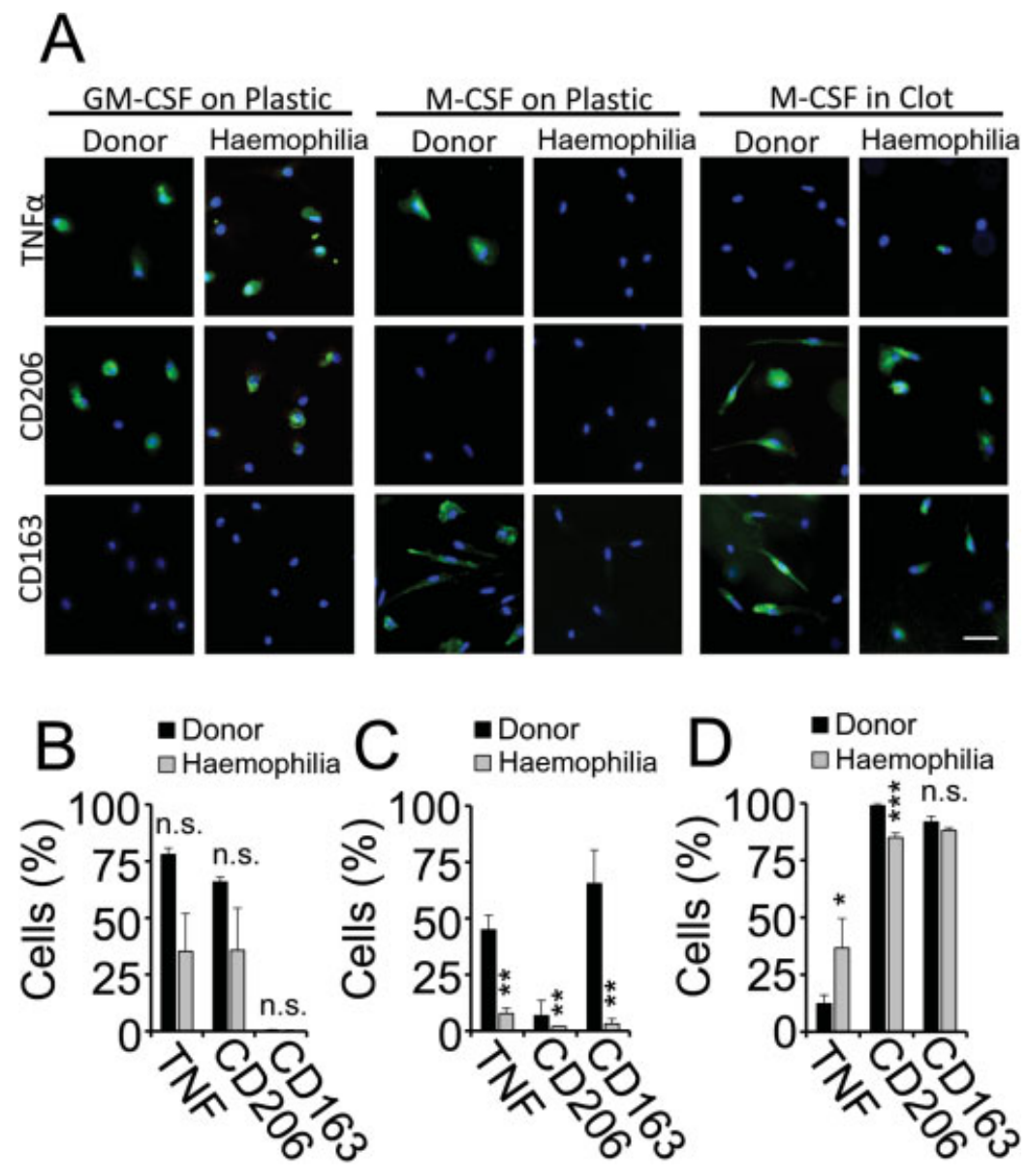

Fig. 6 Macrophages from haemophilia patients exhibit reduced expression of tumour necrosis factor $\alpha$ (TNF $\alpha$ ) and CD163. (A) Macrophages from healthy donors as well as haemophilia patients (ea., $n=2$ ) were isolated with Dynabeads and cultured on conventional cell culture plastic or embedded in clotted plasma in the presence of macrophage colony-stimulating factor (M-CSF) or granulocyte-macrophage colonystimulating factor (GM-CSF). After 7 days, cells were fixed, permeabilized and stained for TNF $\alpha$, CD206 or CD163 (green) and analysed by fluorescence microscopy. Nuclei are stained with 4',6-diamidino-2-phenylindole (DAPI) (blue). Representative fluorescence microscopy images are shown. (B, C) Microscopy fields of plastic attached macrophages were analysed for TNF $\alpha$-, CD206-and CD163-positive cells as per cent of total optical field after treatment with GM-CSF (B) or M-CSF (C). (D) Percentage of TNF $\alpha-$, CD206- and CD163-positive donor and haemophilia macrophages in clotted plasma after treatment with M-CSF. ${ }^{*} p<0.05,{ }^{* *} p<0.01,{ }^{* * *} p<0.001$, n.s., non-significant. Scale bar, 20 mm. 


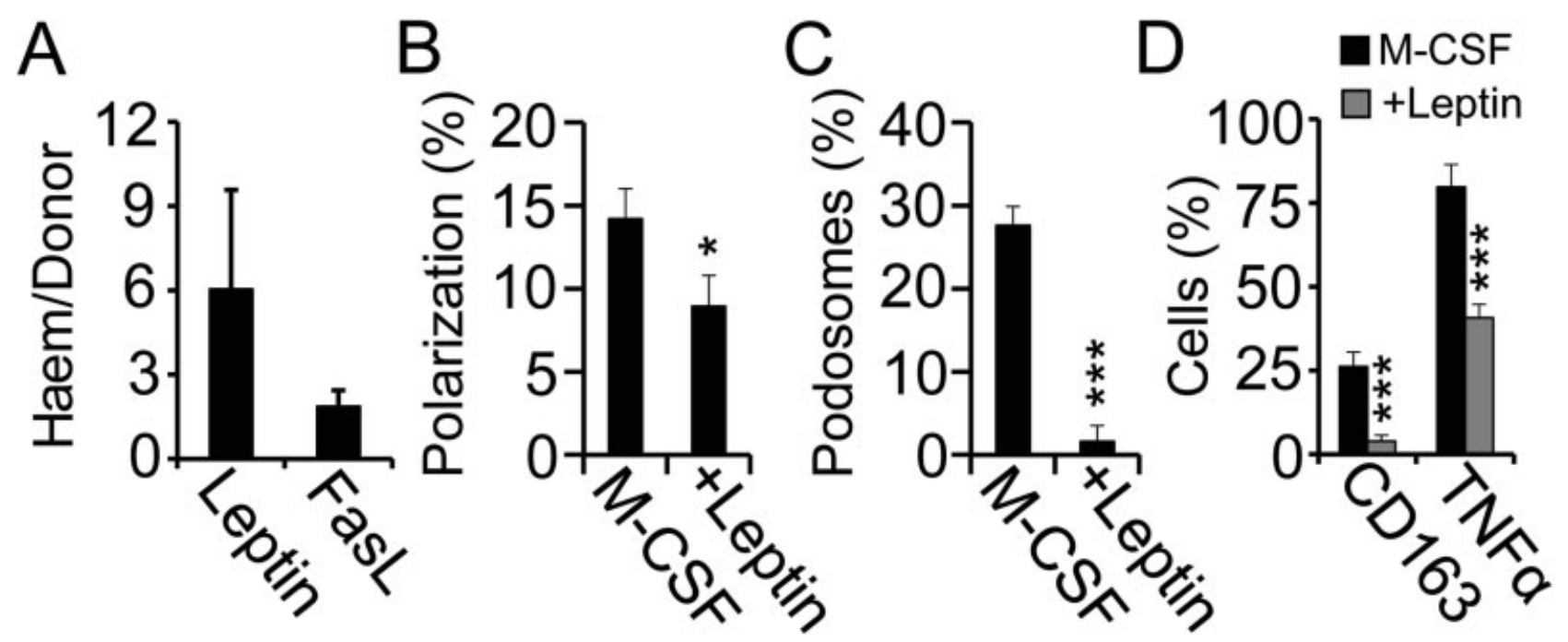

Fig. 7 Leptin restrains macrophage function in haemophilia. (A) Proteins up-regulated in 2 haemophilia patients compared with 2 healthy blood donors (fold increase) after analysis of platelet-poor plasma using the Proteome Profiler Human XL Cytokine Array Kit (R\&D Systems). (B-D) Polarization of THP-1 macrophages on cell culture plastic (B), podosome formation in clotted plasma (C) and expression of CD163 as well as tumour necrosis factor $\alpha$ (TNF $\alpha$ ) expression (D) 96 hours after treatment with macrophage colony-stimulating factor (M-CSF) with or without leptin $(500 \mathrm{ng} / \mathrm{mL}) .{ }^{*} p<0.05,{ }^{* * *} p<0.001$.

\section{CRP and Leptin are Up-Regulated in the Blood Circulation of Haemophiliacs}

Leptin expression has been shown to positively correlate with inflammation as well as body weight. ${ }^{21,22}$ To focus on the link between inflammation and coagulation, we studied the plasma levels of leptin in conjunction with CRP in our main collective of haemophilia patients as well as in a BMIstratified cohort (patients, $n=27$; average BMI, $26.9 \mathrm{~kg} / \mathrm{m}^{2}$; BMI range, 22.4-31.1; donors, $n=38$; average BMI, $26.7 \mathrm{~kg} /$ $\mathrm{m}^{2}$; BMI range, 22.3-31.1) (-Fig. 8A, B). In both cohorts, haemophilia patients exhibited significantly higher plasma leptin levels than the donors and these results were matched by an overall increase in CRP in haemophilia. Sub-group analysis of the BMI-stratified cohort demonstrated that CRP and leptin closely correlated with the severity of disease as both parameters were highest in patients with the lowest residual FVIII or FIX activity ( $\mathbf{- F i g . 8 C}$, D). These differences could not be reproduced when we stratified for patients who receive coagulation factor concentrates on a regular basis (prophylaxis vs. on demand) or patients that were substituted with clotting factors recently and, therefore, had a coagulation factor activity of $>10 \%$ at the time of blood sampling. Moreover, the differences in CRP and leptin were not related to recent bleeding ( $<3$ month), arthropathy or chronic viral infections ( $\mathbf{- F i g . ~ 8 E}, \mathbf{F}$ ). Together, these data demonstrate that CRP and leptin levels are increased in haemophilia patients and, therefore, suggest a link between clotting deficiencies and chronic low-grade inflammation.

\section{Discussion}

The functional coupling between coagulation and inflammation is well established. ${ }^{13-15}$ Here, we show that impaired clotting in haemophilia leads to a deficit in macrophage differentiation, which negatively affects critical regenerative macrophage functions such as wound infiltration and RBC phagocytosis. The altered macrophage function can be traced back to increased levels of CRP and leptin that we detected in the blood circulation of haemophiliacs suggesting that the diminished clotting activity in haemophilia patients is associated with activation of the innate immune system. Moreover, they provide a functional basis for the commonly observed wound healing deficit in haemophilia.

Following their extravasation from the blood circulation, monocytes differentiate into macrophages, which play a central role in orchestrating the response to injury. ${ }^{17}$ In the case of on-going tissue damage or bacterial superinfection, monocytes polarize into M1 macrophages that maintain inflammation by secreting pro-inflammatory cytokines. ${ }^{8,23}$ However, as soon as inflammation subsides macrophages switch into an alternatively activated M2 phenotype, which promotes tissue repair through phagocytosis of neutrophils, RBCs and cell debris as well as the release of regenerative growth factors. ${ }^{24,25}$ This switch occurs typically in response to M2 cytokines such as TGF- $\beta$, IL-4, IL-10 and IL-13. 26,27 However, monocytes can also polarize into M2-like macrophages through the mitogenic activity of M-CSF, a growth factor critical for monocyte and macrophage differentiation. ${ }^{28}$ This process requires no additional M2 cytokines, which underscores the concept that monocytes turn into regenerative macrophages by default as long as no additional inflammatory stimulus is present. ${ }^{28,29}$ Notably, this default mechanism appears to be deregulated in haemophilia monocytes, which spread and polarize only incompletely in response to M-CSF. Moreover, M-CSF fails to properly induce clot invasion and phagocytosis in monocytes from haemophiliacs raising the question whether haemophilia macrophages are competent to fulfil the complex functions that are included in the wound healing response such as wound infiltration and induction of a regenerative tissue response. 

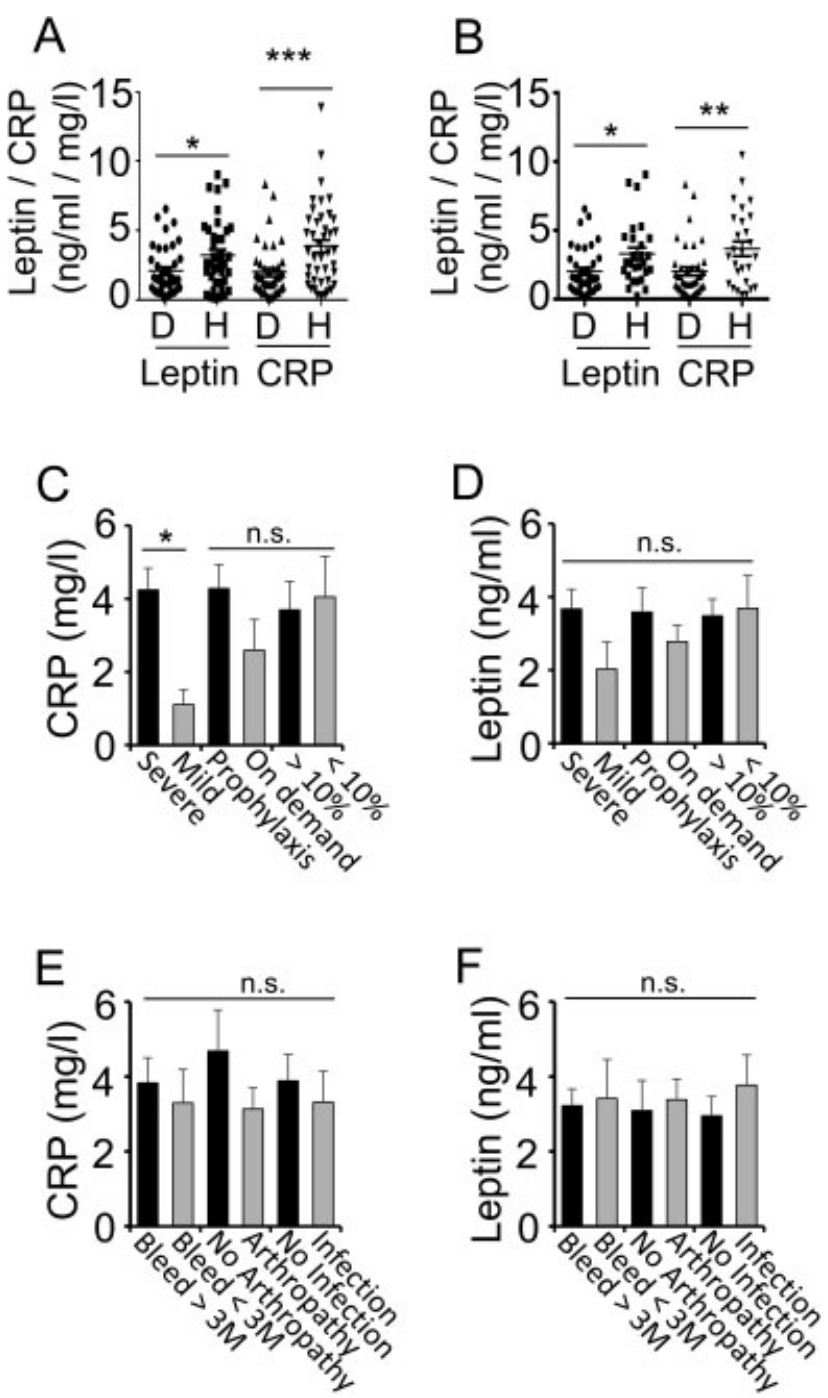

Fig. 8 Plasma leptin and C-reactive protein (CRP) in haemophilia. (A) Leptin $(\mathrm{ng} / \mathrm{mL})$ and CRP $(\mathrm{mg} / \mathrm{L})$ in the plasma from the main patient collective $(\mathrm{H}$, haemophilia; D, donor). (B) Leptin $(\mathrm{ng} / \mathrm{mL})$ and CRP $(\mathrm{mg} / \mathrm{L})$ in a weightcorrected cohort of haemophilia patients $(n=27)$ and healthy blood donors $(n=38)$. (C-F) CRP $(C, E)$ and leptin concentration $(D, F)$ in the plasma of haemophilia patients with severe versus moderate to mild haemophilia, with regular coagulation factor prophylaxis versus on-demand therapy, with effective factor VIII (haemophilia A) or IX (haemophilia B) activity $<$ or $>10 \%$ at the time of blood collection, with bleeding events $<$ or $>3$ months, with or without arthropathy, or with or without chronic viral infections. ${ }^{*} p<0.05$, ${ }^{* *} p<0.01,{ }^{* * *} p<0.001$. n.s., non-significant.

The functional deficiencies of haemophilia macrophages could be traced back to impaired filopodia formation as these actin-rich membrane protrusions represent one of the first steps to activate the invasive and phagocytic machinery of monocytic cells. ${ }^{30}$ The failure of haemophilia macrophages to generate filopodia was detectable after treatment with M-CSF as well as GM-CSF. However, while GM-CSF-treated haemophilia macrophages remained able to express typical activation markers such as TNF $\alpha$ and CD206, this process was largely abated after treatment with M-CSF, which failed to induce TNF $\alpha$ and CD163 in the context of impaired spreading. ${ }^{31-34}$ The deficit in monocyte adhesion is relevant because integrin cell adhesion receptors play an important role in macrophage differentiation by promoting transcription of CSF1-R, the receptor of M-CSF, and by amplifying M-CSF-mediated signals through crosstalk with CSF1-R. ${ }^{35,36}$ This crosstalk appears to be deregulated in haemophilia as demonstrated by the marked reduction of CSF1-R on circulating monocytes in conjunction with reduced cell surface expression of the receptor tyrosine kinase Tie2, which we previously found to be involved in cell spreading and clot invasion..$^{29,37,38}$ In line with this, it has been shown that macrophage recruitment to wounds is impaired in haemophilia B mice, which are similar to macrophage-deficient mice in that they exhibit deficits in wound healing. ${ }^{2,25,39}$ However, while the deficiency in macrophage-depleted mice is lasting, haemophilia mice rather exhibit a delay in monocyte recruitment since macrophages start to populate wounds at a later time point. This observation could be owed to reduced invasion in absence of proper clotting or, alternatively, to the diminished clot invasiveness of a distinctive monocyte phenotype similar to the one we identified in our patients, which largely fails to invade clotted plasma.

The incapacity to interact with blood clot has not only consequences for the recruitment of macrophages but also for their function, which is known to be highly contextdependent. ${ }^{40}$ Accordingly, we show here that clot-embedded donor macrophages suppress the expression of the proinflammatory cytokine TNF $\alpha$ and promote expression of the anti-inflammatory receptor CD206 in response to MCSF after embedding in clotted plasma from healthy donors. Therefore, these data suggest that blood clot not only is instrumental in recruiting monocytes but also in providing an environment that promotes differentiation of regenerative macrophages. Notably, the regenerative potential of clotted plasma was also effective on haemophilia macrophages, which regained the capacity to at least partially differentiate in response to M-CSF after failing to do so on plastic. One possible explanation for the corrective potential of the plasma clot could be the presence of thrombin, which was added to the monocyte suspension to cleave fibrinogen and, therefore, generate fibrin. This aspect seems particularly relevant since we found that thrombin can promote M-CSFmediated macrophage polarization. However, there is also ample precedence that clotted plasma can modulate inflammation through adhesive interactions with different classes of fibrin(ogen)- and fibronectin-binding integrins on monocytes as well as macrophages. ${ }^{12,41,42}$

Our data confirm a direct effect of the clotting system on the function of macrophages. To further analyse the systemic aspects of the innate immunity in haemophilia, we performed a proteomics screen, which showed marked up-regulation of the immune modulatory cytokine leptin, which becomes released from adipose tissue in obesity and after inflammation. $^{21,43}$ Significantly, treating THP-1 monocytes with recombinant leptin reiterates the specific functional and phenotypical alterations that we observed in haemophilia macrophages. This link was further solidified by demonstrating that leptin is significantly increased in a BMI-corrected cohort of haemophilia patients that at the same time harbour increased plasma levels of CRP, which suggests the presence of chronic low-grade inflammation similar to what has been described for patients 
with obesity, cardiovascular disease or type 2 diabetes. ${ }^{44-46}$ Sub-group analysis revealed low FVIII/FIX activity as the critical determinant for the increased release of CRP and leptin, while complications such as bleeding, arthropathy or chronic viral infections played no or only minor roles. Based on these data, a concept is emerging wherein haemophilia patients as a result of their clotting defect experience increased inflammation, which is associated with an acute phase reaction and deregulation of innate immune cells. These changes could aggravate typical complication of haemophilia such as impaired wound healing as well as joint inflammation. Moreover, they may offset the beneficial effects of clotting impairment for the development of cardiovascular disease in aging haemophiliacs, which strongly correlates with increased CRP. ${ }^{47}$ The immune modulatory changes we describe in haemophilia patients appear to be multi-factorial and, therefore, are not easily remedied by a short-lived increase of clotting factor activity as is the case for most established prophylaxis regiments. Therefore, CRP and leptin may be useful parameters to evaluate the innate immune response of haemophilia patients in general, but also in the context of novel treatment modalities that may provide a longer lasting correction of the underlying clotting impairment.

\section{What is known about this topic?}

- Haemophilia is associated with deficient wound healing and inflammatory joint disease.

- Macrophages contribute to wound healing as well as inflammation.

- Coagulation factor deficiencies cause deregulation of monocyte adhesion and signaling.

\section{What does this paper add?}

- Monocyte differentiation is deregulated in haemophilia causing deficits in macrophage functions correlating with tissue regeneration and wound healing.

- The functional deficits of haemophilia macrophages can be traced back to increased levels of C-reactive protein and leptin in the blood circulation of haemophiliacs.

- The diminished clotting activity in haemophilia patients appears to be directly linked to the activation of the innate immune response.

Authors' Contributions

L.M. Knowles, H. Eichler and J. Pilch designed the research; L.M. Knowles, D. Lessig and M. Bernard performed the experiments; E. Schwarz contributed to methods and reagents; L.M. Knowles and J. Pilch analysed the data; and L.M. Knowles and J. Pilch wrote the manuscript.

\section{Funding}

This work was supported by CSL Behring GmbH (J.P. and H.E.), the Pfizer ASPIRE Award (J.P.) and the Hans Egli Stipend of the Gesellschaft für Thrombose- und Hämostaseforschung e.V. (L.M.K.).
Conflict of Interest

None declared.

\section{References}

1 Monroe DM, Hoffman M. The clotting system - a major player in wound healing. Haemophilia 2012;18(Suppl 5):11-16

2 Hoffman M, Harger A, Lenkowski A, Hedner U, Roberts HR, Monroe DM. Cutaneous wound healing is impaired in hemophilia B. Blood 2006;108(09):3053-3060

3 Aronstam A, Rainsford SG, Painter MJ. Patterns of bleeding in adolescents with severe haemophilia A. BMJ 1979;1(6161):469-470

4 Hoots WK. Pathogenesis of hemophilic arthropathy. Semin Hematol 2006;43(01, Suppl 1):S18-S22

5 Oldenburg J, Zimmermann R, Katsarou O, et al; Cross-sectional MRI study investigators. Controlled, cross-sectional MRI evaluation of joint status in severe haemophilia A patients treated with prophylaxis vs. on demand. Haemophilia 2015;21(02):171-179

6 Morris CJ, Blake DR, Wainwright AC, Steven MM. Relationship between iron deposits and tissue damage in the synovium: an ultrastructural study. Ann Rheum Dis 1986;45(01):21-26

7 Roosendaal G, Vianen ME, Wenting MJ, et al. Iron deposits and catabolic properties of synovial tissue from patients with haemophilia. J Bone Joint Surg Br 1998;80(03):540-545

8 Sen D, Chapla A, Walter N, Daniel V, Srivastava A, Jayandharan GR. Nuclear factor (NF)-KB and its associated pathways are major molecular regulators of blood-induced joint damage in a murine model of hemophilia. J Thromb Haemost 2013;11(02): 293-306

9 Nieuwenhuizen L, Schutgens RE, Coeleveld K, et al. Hemarthrosis in hemophilic mice results in alterations in M1-M2 monocyte/ macrophage polarization. Thromb Res 2014;133(03):390-395

10 Hoppstädter J, Seif M, Dembek A, et al. M2 polarization enhances silica nanoparticle uptake by macrophages. Front Pharmacol 2015;6:55

11 Steinbrecher KA, Horowitz NA, Blevins EA, et al. Colitis-associated cancer is dependent on the interplay between the hemostatic and inflammatory systems and supported by integrin alpha(M)beta(2) engagement of fibrinogen. Cancer Res 2010;70(07):2634-2643

12 Kopec AK, Abrahams SR, Thornton S, et al. Thrombin promotes diet-induced obesity through fibrin-driven inflammation. J Clin Invest 2017;127(08):3152-3166

13 von Brühl ML, Stark K, Steinhart A, et al. Monocytes, neutrophils, and platelets cooperate to initiate and propagate venous thrombosis in mice in vivo. J Exp Med 2012;209(04):819-835

14 Rathnakumar K, Savant S, Giri H, et al. Angiopoietin-2 mediates thrombin-induced monocyte adhesion and endothelial permeability. J Thromb Haemost 2016;14(08):1655-1667

15 Riewald M, Petrovan RJ, Donner A, Mueller BM, Ruf W. Activation of endothelial cell protease activated receptor 1 by the protein $C$ pathway. Science 2002;296(5574):1880-1882

16 Kopec AK, Joshi N, Cline-Fedewa H, et al. Fibrin(ogen) drives repair after acetaminophen-induced liver injury via leukocyte $\alpha_{M} \beta_{2}$ integrin-dependent upregulation of Mmp12. J Hepatol 2017;66(04):787-797

17 Shi C, Pamer EG. Monocyte recruitment during infection and inflammation. Nat Rev Immunol 2011;11(11):762-774

18 Park H, Ishihara D, Cox D. Regulation of tyrosine phosphorylation in macrophage phagocytosis and chemotaxis. Arch Biochem Biophys 2011;510(02):101-111

19 McWhorter FY, Wang T, Nguyen P, Chung T, Liu WF. Modulation of macrophage phenotype by cell shape. Proc Natl Acad Sci U S A 2013;110(43):17253-17258

20 Masters TA, Pontes B, Viasnoff V, Li Y, Gauthier NC. Plasma membrane tension orchestrates membrane trafficking, cytoskeletal remodeling, and biochemical signaling during phagocytosis. Proc Natl Acad Sci U S A 2013;110(29):11875-11880 
21 Grunfeld C, Zhao C, Fuller J, et al. Endotoxin and cytokines induce expression of leptin, the ob gene product, in hamsters. J Clin Invest 1996;97(09):2152-2157

22 Maffei M, Halaas J, Ravussin E, et al. Leptin levels in human and rodent: measurement of plasma leptin and ob RNA in obese and weight-reduced subjects. Nat Med 1995;1(11):1155-1161

23 Mosser DM, Edwards JP. Exploring the full spectrum of macrophage activation. Nat Rev Immunol 2008;8(12):958-969

24 Fadok VA, Bratton DL, Konowal A, Freed PW, Westcott JY, Henson PM. Macrophages that have ingested apoptotic cells in vitro inhibit proinflammatory cytokine production through autocrine/paracrine mechanisms involving TGF-beta, PGE2, and PAF. J Clin Invest 1998;101(04):890-898

25 Mirza R, DiPietro LA, Koh TJ. Selective and specific macrophage ablation is detrimental to wound healing in mice. Am J Pathol 2009;175(06):2454-2462

26 Hart PH, Vitti GF, Burgess DR, Whitty GA, Piccoli DS, Hamilton JA. Potential antiinflammatory effects of interleukin 4: suppression of human monocyte tumor necrosis factor alpha, interleukin 1 , and prostaglandin E2. Proc Natl Acad Sci U S A 1989;86(10): 3803-3807

27 Bogdan C, Paik J, Vodovotz Y, Nathan C. Contrasting mechanisms for suppression of macrophage cytokine release by transforming growth factor-beta and interleukin-10.J Biol Chem 1992;267(32): 23301-23308

28 Fleetwood AJ, Lawrence T, Hamilton JA, Cook AD. Granulocytemacrophage colony-stimulating factor (CSF) and macrophage CSF-dependent macrophage phenotypes display differences in cytokine profiles and transcription factor activities: implications for CSF blockade in inflammation. J Immunol 2007;178(08): 5245-5252

29 Forget MA, Voorhees JL, Cole SL, et al. Macrophage colonystimulating factor augments Tie2-expressing monocyte differentiation, angiogenic function, and recruitment in a mouse model of breast cancer. PLoS One 2014;9(06):e98623

30 Kress H, Stelzer EH, Holzer D, Buss F, Griffiths G, Rohrbach A. Filopodia act as phagocytic tentacles and pull with discrete steps and a load-dependent velocity. Proc Natl Acad Sci U S A 2007;104 (28):11633-11638

31 Buechler C, Ritter M, Orsó E, Langmann T, Klucken J, Schmitz G. Regulation of scavenger receptor CD163 expression in human monocytes and macrophages by pro- and antiinflammatory stimuli. J Leukoc Biol 2000;67(01):97-103

32 Lacey DC, Achuthan A, Fleetwood AJ, et al. Defining GM-CSF- and macrophage-CSF-dependent macrophage responses by in vitro models. J Immunol 2012;188(11):5752-5765

33 Neu C, Sedlag A, Bayer C, et al. CD14-dependent monocyte isolation enhances phagocytosis of listeria monocytogenes by proinflammatory, GM-CSF-derived macrophages. PLoS One 2013; 8(06): e66898

34 Boocock CA, Jones GE, Stanley ER, Pollard JW. Colony-stimulating factor-1 induces rapid behavioural responses in the mouse macrophage cell line, BAC1.2F5. J Cell Sci 1989;93(Pt 3):447-456

35 Shi $C$, Zhang $X$, Chen $Z$, et al. Integrin engagement regulates monocyte differentiation through the forkhead transcription factor Foxp1. J Clin Invest 2004;114(03):408-418

36 Joshi S, Singh AR, Zulcic M, et al. Rac2 controls tumor growth, metastasis and M1-M2 macrophage differentiation in vivo. PLoS One 2014;9(04):e95893

37 Knowles LM, Malik G, Pilch J. Plasma fibronectin promotes tumor cell survival and invasion through regulation of Tie2. J Cancer 2013;4(05):383-390

38 Tippett E, Cheng WJ, Westhorpe C, et al. Differential expression of CD163 on monocyte subsets in healthy and HIV-1 infected individuals. PLoS One 2011;6(05):e19968

39 Goren I, Allmann N, Yogev N, et al. A transgenic mouse model of inducible macrophage depletion: effects of diphtheria toxindriven lysozyme M-specific cell lineage ablation on wound inflammatory, angiogenic, and contractive processes. Am J Pathol 2009;175(01):132-147

40 Mantovani A, Biswas SK, Galdiero MR, Sica A, Locati M. Macrophage plasticity and polarization in tissue repair and remodelling. J Pathol 2013;229(02):176-185

41 Flick MJ, Lajeunesse CM, Talmage KE, et al. Fibrin(ogen) exacerbates inflammatory joint disease through a mechanism linked to the integrin alphaMbeta2 binding motif. J Clin Invest 2007;117 (11):3224-3235

42 Laouar A, Collart FR, Chubb CB, Xie B, Huberman E. Interaction between alpha 5 beta 1 integrin and secreted fibronectin is involved in macrophage differentiation of human HL-60 myeloid leukemia cells. J Immunol 1999;162(01):407-414

43 Friedman JM, Halaas JL. Leptin and the regulation of body weight in mammals. Nature 1998;395(6704):763-770

44 Lagrand WK, Visser CA, Hermens WT, et al. C-reactive protein as a cardiovascular risk factor: more than an epiphenomenon? Circulation 1999;100(01):96-102

45 Visser M, Bouter LM, McQuillan GM, Wener MH, Harris TB. Elevated C-reactive protein levels in overweight and obese adults. JAMA 1999;282(22):2131-2135

46 Cardoso CR, Leite NC, Salles GF. Prognostic importance of Creactive protein in high cardiovascular risk patients with type 2 diabetes mellitus: the Rio de Janeiro Type 2 Diabetes Cohort Study. J Am Heart Assoc 2016;5(11):e004554

47 Sood SL, Cheng D, Ragni M, et al. A cross-sectional analysis of cardiovascular disease in the hemophilia population. Blood Adv 2018;2(11):1325-1333 